\title{
Analysis of the tonic vibration reflex: influence of vibration variables on motor unit synchronization and fatigue
}

\begin{abstract}
The influence of vibration frequency (40, 80, $100,120,150$, or $200 \mathrm{~Hz}$ ) at selected displacement amplitudes $(0.2,0.3 \mathrm{~mm})$ on tonic vibration reflex (TVR) characteristics was investigated. The degree of synchronization of motor unit activity with vibratory stimuli in ten humans was determined using the electromyographic (EMG) activity of the finger and wrist flexor muscles when vibration was applied to the distal tendons of the hand flexor muscles. The EMG spectral analysis indicates that harmonic and subharmonic motor unit synchronization mechanisms contribute to the modulation of the amplitude of the TVR as the vibration frequency increases. Harmonic synchronization decreases while subharmonic synchronization increases as vibration frequency increases. It is suggested that the synchronization process influences muscle fatigue, since it forces the driving of motor units, leading to a decrease in contraction efficiency. This phenomenon most probably results from an impairment of excitation-contraction coupling. High-frequency vibration $(>150 \mathrm{~Hz})$ tends to induce less motor unit synchronization in a frequency range beyond the known mechanical resonance of biological tissues. The findings of this study may be applied to the design of hand-held power tools, since their vibration triggers the TVR in active muscles.
\end{abstract}

Key words Electromyography - Spinal reflexes · Flexor carpi radialis · Flexor digitorum profundus . Human

B.J. Martin $(\square)$

Dept. of Industrial and Operations Engineering,

Center for Ergonomics, The University of Michigan,

1205 Beal avenue, Ann Arbor, MI 40109-2117, USA

H.-S. Park

Dept. of Industrial Engineering, HongIk University, Sangsu-Dong 72-1, Mapo-Ku, Seoul, Korea 121-791

\section{Introduction}

It is well known that mechanical vibration applied to the muscle belly or tendon can elicit a reflex muscle contraction (Hagbarth and Eklund 1965). This response, named the tonic vibration reflex (TVR), results mainly from the vibration-induced activity of the muscle spindle Ia fibres (De Gail et al. 1966; Burke et al. 1976a; Roll et al. 1989), and is mediated by monosynaptic and polysynaptic pathways (Matthews 1966; Desmedt and Godaux 1980; Romaiguère et al. 1991).

The relative roles of the two pathways are of concern in the study of motor control and the relationship between the TVR, muscle fatigue, and stress. The TVR may contribute to muscle fatigue and/or increase the risk of cumulative trauma disorders observed after repetitive exposure of the hand to vibration (Park and Martin 1993). In this type of vibration exposure, the TVR superimposed on ongoing voluntary muscle activity should be minimized or eliminated. Several studies have indicated that the TVR is composed of motor unit activity synchronized and unsynchronized with the vibration cycle (Homma et al. 1972; Hori et al. 1989; Romaiguère et al. 1991; Lebedev and Polyakov 1992). Further description of the TVR, and the behavior of motor unit activity as a function of the vibratory stimulus variables should lead to a better understanding of the reflex responses of proprioceptive origin and the mechanisms of the risks associated with vibration exposure. Risk factors concerning soft tissue disorders include vibration variables and force exertion, which influence vibration transmissibility to the human body (Pyykkö et al. 1976; Reynolds 1977).

In the present study, changes in the TVR and motor unit synchronization in the finger and wrist flexor muscles were analyzed as a function of the frequency and two displacement amplitudes of vibration applied to their distal tendons, and as a function of the initial level of voluntary muscle contraction. 


\section{Methods}

\section{Subjects}

Ten healthy subjects participated in the experiment as paid volunteers and gave informed consent. Their average age was 22.6 years. All of the subjects were free from any known neurological or musculoskeletal disorders. The experiment was approved by the ethics committee of University of Michigan School of Public Health.

\section{Mechanical stimulation}

The methods have been presented previously (Park and Martin 1993). The subjects were comfortably seated. The right hand, in slight extension, gripped a "vertical" handle $\left(80^{\circ}\right)$ fixed to a padded arm-rest (Fig. 1). An adjustable support was used to maintain the wrist in this position and thus forced isometric conditions. The handle angle was chosen to obtain a comfortable arm posture and an advantageous position of the forearm for tendon vibration and electromyographic (EMG) recording. The handle was equipped with a strain gauge dynamometer. The height of the arm-rest and the horizontal position of the handle were adjusted to obtain approximately a $120^{\circ}$ angle at the elbow.

Vibration was applied perpendicularly to the distal tendons of the hand flexor muscles by means of an electromagnetic vibrator (Ling Dynamic System, 203) equipped with a specially designed probe. An accelerometer placed inside the vibrating probe provided feedback to a servo controller (compressor: Trig-tek $801 \mathrm{~B}$; monitor: Trig-tek 610B) driven by a sine wave generator. The servocontrolled vibration signal was transmitted to the vibrator through a power amplifier. Over the tested frequency range, the system maintained a constant displacement amplitude of the probe throughout the vibration period, regardless of the tendon tension. The vibrating probe was pressed on the tendons to obtain a $1-\mathrm{mm}$ recession of the probe, which produced an initial force of about $4 \mathrm{~N}$ (stiffness of the vibrator suspension guidance: $4 \mathrm{~N} \cdot \mathrm{mm}^{-1}$. Ling Dynamic System, 203). Marks engraved on the probe were used to determine the displacement of the probe. The sharp edge of the vibrator housing was used as a reference line. Vibration variables and the initial deformation of the tendon were carefully controlled, as mechanical characteristics of the stimulus (Cordo et al. 1993) and muscle tension (Matthews 1966; Cordo et al. 1993) influence the response of muscle stretch receptors. Vibration frequencies $(40$, $80,100,120,150$, or $200 \mathrm{~Hz}$ ) with constant peak-to-peak displacement amplitudes $(0.2$ and $0.3 \mathrm{~mm})$ were applied to the flexor tendon group.

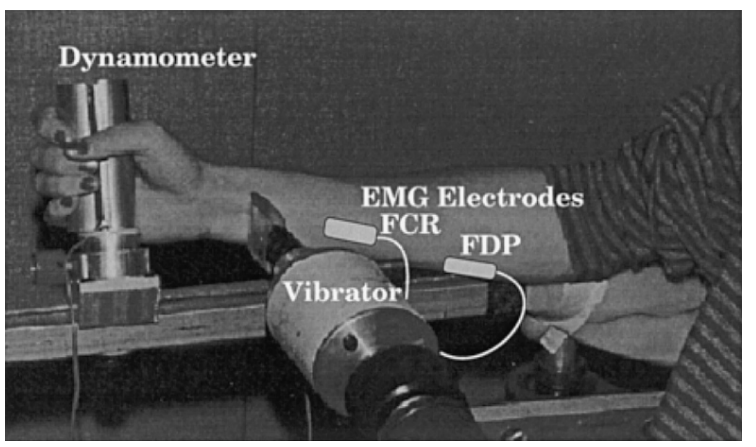

Fig. 1 Experimental set-up. Vibration is applied perpendicularly to the distal flexor tendons. Preamplified surface electrodes are used to record the EMG activity of the flexor carpi radialis $(F C R)$ and flexor digitorum profundus $(F D P)$ muscles. The grip force is measured by a strain guage dynamometer

\section{EMG recording sites}

The EMGs of the flexor digitorum profundus (FDP), the flexor carpi radialis (FCR), and extensor digitorum communis (EDC) were recorded by pairs of small cupular surface electrodes contained in preamplifier devices. The inter-electrode distance was 2 $\mathrm{cm}$. A ground electrode was attached at the radial styloid of the wrist. After localization of the muscles by palpation and resistive maneuvers, optimum electrode placements were obtained by successive trial and error (with the forearm held in the position of the experimental situation while EMG signals were displayed on an oscilloscope) in order to minimize cross-talk. For the FCR and EDC the electrodes were placed on the bellies of the respective muscles. For the FDP, the electrodes were placed at a location about one-third of the distance between the olecranon and the ulnar styloid, and $1 \mathrm{~cm}$ medial to the ulna shaft; at this location, this muscle lies just below the thin aponeurosis of the flexor carpi ulnaris. The EDC activity was recorded to observe the reciprocal inhibition and to ascertain that the TVR did not switch to an "antagonist vibration response" (Roll et al. 1980) during the experiment and, more specifically, in the resting condition.

\section{Experimental procedure}

The level of maximal voluntary contraction (MVC) of the grip was determined before each experiment. The subjects were required to gradually increase their grip exertion up to the maximum level within $2 \mathrm{~s}$, and maintain the level for $3 \mathrm{~s}$. The MVC was obtained from the mean of the peaks of two replicate procedures separated by a 5 -min rest period. The subjects were then trained to maintain a grip force of $10 \%$ and $20 \%$ of their MVC for 1-min periods, using only their own intrinsic proprioceptive (and exteroceptive) feedback. The muscle contraction levels were selected to represent grip exertions during the operation of small power hand-tools (Radwin et al. 1987). At first, visual feedback from a voltmeter connected to the dynamometer was provided. After the subject felt familiar with the required submaximal level of contraction, the visual feedback was gradually suppressed and replaced by oral information. The test session started only after the grip performance had reached a steady state and varied less than $4 \%$.

For each trial, the subject started to exert one of three different grip forces $(0,10$ or $20 \%$ of the MVC), while viewing the voltmeter ( $0 \%$ MVC corresponds to a resting situation in which the fingers are wrapped around the handle without exerting significant force). Once the proper level of force was reached the visual feedback was suppressed and oral feedback was given by the experimenter until the $\% \mathrm{MVC}$ level was stabilized and the subject felt ready for the trial to begin. Force stabilization was reached within $5-10 \mathrm{~s}$. No external feedback was provided during the trial.

Data were collected after force stabilization while the subject maintained the submaximal level of contraction for $60 \mathrm{~s}$ consisting of a 15-s control period followed by a 45 -s period during which vibration was applied continuously. The vibration frequency was varied randomly across the contraction levels for constant peak-topeak vibration displacement amplitudes of 0.2 and $0.3 \mathrm{~mm}$.

For each subject, the experiment was carried out over 2 days to reduce any possible effect of boredom and fatigue. Data were collected over a total of 36 trials for each subject (3 levels of contraction $\times 6$ frequencies $\times 2$ displacement amplitudes). Each subject served as their own control. Due to the practical limitations imposed by the setting of the vibration displacement amplitude, trials for each subject were randomized across the frequencies and contraction levels. The order of presentation of displacement amplitudes was randomized across subjects. A 2-min rest period separated each consecutive trial.

In a complementary experiment performed by five subjects an isometric grip exertion ( $20 \%$ MVC) was maintained for 8 min with or without tendon vibration $(100 \mathrm{~Hz}, 0.3 \mathrm{~mm})$ in the situation described above. 


\section{Data recording and processing}

The EMGs of the ECR, FDP, and the FCR muscles were recorded simultaneously. The built-in preamplifier minimized noise and artifacts of electrical and mechanical origins. The respective signals were then amplified, and processed by an electronic circuit to obtain the rms values. For the raw EMG, the signals were low-pass filtered at $300 \mathrm{~Hz}$ for anti-aliasing purposes. Raw EMGs, rmsEMGs and grip force signals were sampled at $1 \mathrm{kHz}$ for $60 \mathrm{~s}$, as

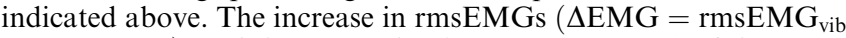
$-\mathrm{rmsEMG}_{\mathrm{ref}}$ ), and the power density spectrum (PDS) of the EMG signals computed for the last $15 \mathrm{~s}$ of the vibration period were analyzed as a function of initial contraction levels and vibration frequencies for both displacement amplitudes. A total of 15 periodograms of $1024 \mathrm{~ms}$ covering the $0-$ to $256-\mathrm{Hz}$ frequency band with $1-\mathrm{Hz}$ resolution were averaged; a Hanning window was used. To quantify motor unit synchronization, the area of spectral peaks at the vibration frequency and at the first subharmonic frequency were expressed as percentages of the total power of the PDS. The normalized synchronization index (SYNC) at the vibration frequency (VF) or subharmonic frequency (SF) was defined as:

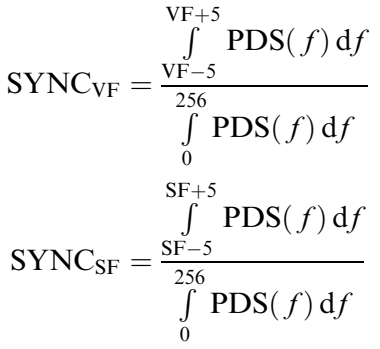

The absence of artifacts was systematically verified before each experiment. A spectral analysis of all EMG signals was performed in passive (resting) and active (voluntary contraction) conditions while vibration was turned on. For each condition, the probe was placed close to, but not in contact with, the muscle tendons, or was applied to several areas away from the tendons. The PDSs were "flat" while resting and did not exhibit "prominent peaks" correlated with the vibratory stimulus during voluntary contraction.

Data analysis

Repeated measures analysis of variance (ANOVA) treating the subject as a random blocking factor was performed on the average $\triangle \mathrm{EMG}, \mathrm{SYNC}_{\mathrm{VF}}$, and $\mathrm{SYNC}_{\mathrm{SF}}$ to determine the effects of the vibration variables and initial muscle contraction on the TVR and motor unit synchronization.

\section{Results}

Spectral analysis

The spectral analysis showed a correlation between the vibratory stimulus and motor unit activity. It should be stressed that spectral peaks that are correlated with vibration result from the phase shift of a number of synchronized motor units distributed along the vibration cycle. Narrow peaks at the vibration frequency and/or subharmonic frequency were present in the PDS of the respective EMG signals. Examples of typical PDSs of the FCR EMG, obtained from one subject under two conditions, are shown in Fig. 2. For a $100 \mathrm{~Hz}$ vibration (Fig. 2, upper panel), a high peak was observed at $100 \mathrm{~Hz}$, indicating strong synchronization of motor unit
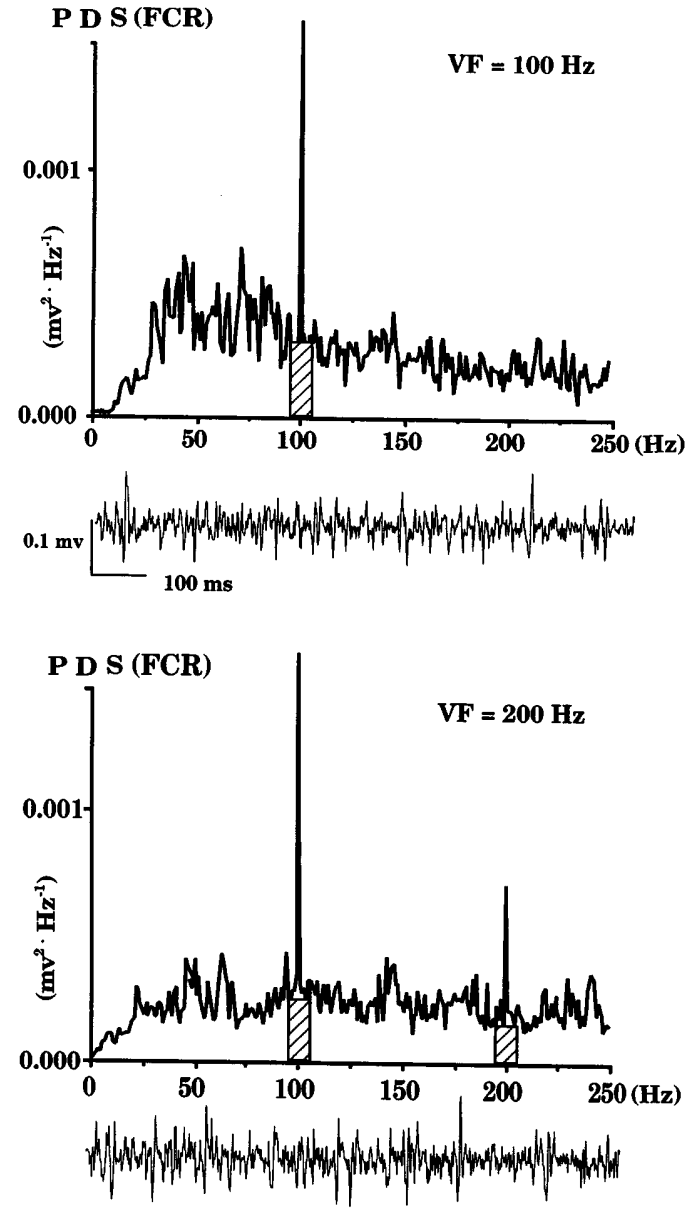

Fig. 2 Representative examples of FCR EMG power density spectra $(P D S)$ showing the influence of vibration frequency on the synchronization process $(100 \mathrm{~Hz}$, upper panel; $200 \mathrm{~Hz}$, lower panel). A fragment of the corresponding EMG is presented below each spectrum. Each spectrum corresponds to the average of 15 periodograms of $1024 \mathrm{~ms}$, obtained for one subject. The hatched areas represent the $10 \mathrm{~Hz}$ bandwidths used to compute synchronization indices. The spectral peak of the harmonic component is predominant for the $100 \mathrm{~Hz}$ vibration while the subharmonic peak is predominant for the $200 \mathrm{~Hz}$ vibration. (VF Vibration frequency)

activity with the vibration frequency. For a $200 \mathrm{~Hz}$ vibration (Fig. 2, lower panel), the main peak was observed at the subharmonic frequency $(100 \mathrm{~Hz})$ while a smaller peak was observed at the vibration frequency $(200 \mathrm{~Hz})$. This inversion of the relative amplitude of the respective peaks indicates that subharmonic synchronization is predominant for vibration in the high frequency range, which is quantified by averaged data presented below.

The results of the ANOVA of the average indices (Table 1) indicate that the tested vibration amplitudes had no effect on any of the indices, while the effect of vibration frequency was significant in all cases, and the effect of initial muscle contraction was significant in all cases except for the synchronization at the vibration frequency $\left(\mathrm{SYNC}_{\mathrm{VF}}\right)$ for the FDP. 
Table 1 ANOVA results for the motor until synchronization. ( $V F$ Vibration frequency, $S Y N C$ normalized synchronization index)

\footnotetext{
* Significant, $\alpha=0.05$
}

\begin{tabular}{|c|c|c|c|c|c|}
\hline \multirow[t]{3}{*}{ Effect } & \multirow[t]{3}{*}{ d } & \multicolumn{4}{|l|}{$P$ values } \\
\hline & & \multicolumn{2}{|c|}{ Finger flexor } & \multicolumn{2}{|c|}{ Wrist flexor } \\
\hline & & $\mathrm{SYNC}_{\mathrm{VF}}$ & $\mathrm{SYNC}_{\mathrm{SF}}$ & $\mathrm{SYNC}_{\mathrm{VF}}$ & $\mathrm{SYNC}_{\mathrm{SF}}$ \\
\hline Frequency (VF) & 5 & $0+{ }^{*}$ & $0+{ }^{*}$ & $0+{ }^{*}$ & $0+{ }^{*}$ \\
\hline Displacement (D) & 1 & 0.165 & 0.745 & 0.210 & 0.837 \\
\hline Contraction (IC) & 2 & 0.483 & $0.002^{*}$ & $0.012^{*}$ & $0+{ }^{*}$ \\
\hline $\mathrm{VF} \times \mathrm{D}$ & 5 & 0.602 & $0.014^{*}$ & 0.419 & $0.001^{*}$ \\
\hline $\mathrm{VF} \times \mathrm{IC}$ & 10 & $0+{ }^{*}$ & 0.435 & $0.011_{*}^{*}$ & $0.003^{*}$ \\
\hline $\mathrm{D} \times \mathrm{IC}$ & 2 & 0.471 & 0.854 & $0.017^{*}$ & 0.375 \\
\hline $\mathrm{VF} \times \mathrm{D} \times \mathrm{IC}$ & 10 & 0.873 & 0.969 & 0.353 & $0.001^{*}$ \\
\hline
\end{tabular}

Vibration displacement amplitude

It is assumed that the TVR was stabilized (steady state) during the last $15 \mathrm{~s}$ of the vibration period and that most of the primary endings were recruited even at the lower level of vibration amplitude (Roll et al. 1989). Thus, further recruitment of motor units with a small increment in vibration amplitude was limited. Since this variable was not significant (Table 1), the indices were then summed across the amplitudes and the subjects, and analyzed as a function of vibration frequency for each initial contraction.

\section{Vibration frequency}

The graphs in Fig. 3 show, for both muscles at each initial contraction, that as vibration frequency increases the normalized synchronization index $\mathrm{SYNC}_{\mathrm{VF}}$ decreases while the $\mathrm{SYNC}_{\mathrm{SF}}$ increases. However, an exception was observed for the FDP between 40 and $80 \mathrm{~Hz}$ in the resting condition. Beyond $100 \mathrm{~Hz}, \mathrm{SYNC}_{\mathrm{SF}}$ becomes predominant (paired $t$-test, $P<0.05$ ). A "cut off" frequency (intersection of the harmonic and subharmonic responses) appears located above $100 \mathrm{~Hz}$. This observation is supported by the Tukey comparisons, which indicate that there was a significant difference in SYNC $_{\mathrm{VF}}$ either between $80 \mathrm{~Hz}$ and $100 \mathrm{~Hz}$ or between $100 \mathrm{~Hz}$ and $120 \mathrm{~Hz}$ for both muscles. These results are compatible with those relating to the strength of the TVR, as presented in Fig. 5 (see Discussion).

\section{Initial muscle contraction level}

When compared to the resting condition ( $0 \% \mathrm{MVC})$, the initial contraction of $10 \%$ MVC resulted in a significant $(P<0.05)$ increase of the $\mathrm{SYNC}_{\mathrm{VF}}$ for the FCR, and of the $\mathrm{SYNC}_{\mathrm{SF}}$ for both flexor muscles (Fig. 3). The increase in harmonic synchronization $\left(\mathrm{SYNC}_{\mathrm{VF}}\right)$ is observed at $40 \mathrm{~Hz}$ essentially for the FDP and at up to $100 \mathrm{~Hz}$ for the FCR, while non-significant changes occur beyond $120 \mathrm{~Hz}$ for both muscles. The increase in subharmonic synchronization $\left(\mathrm{SYNC}_{\mathrm{SF}}\right)$ is marked at all frequencies except $40 \mathrm{~Hz}$ for both muscles. The initial contraction of $20 \%$ MVC induced similar effects; however, no significant differences in the synchronization indices were observed between $10 \%$ and $20 \%$ MVC.

Muscle

The synchronization patterns of the motor unit activity of both flexor muscles were similar. However, a paired $t$-test revealed that the magnitude of motor unit activity
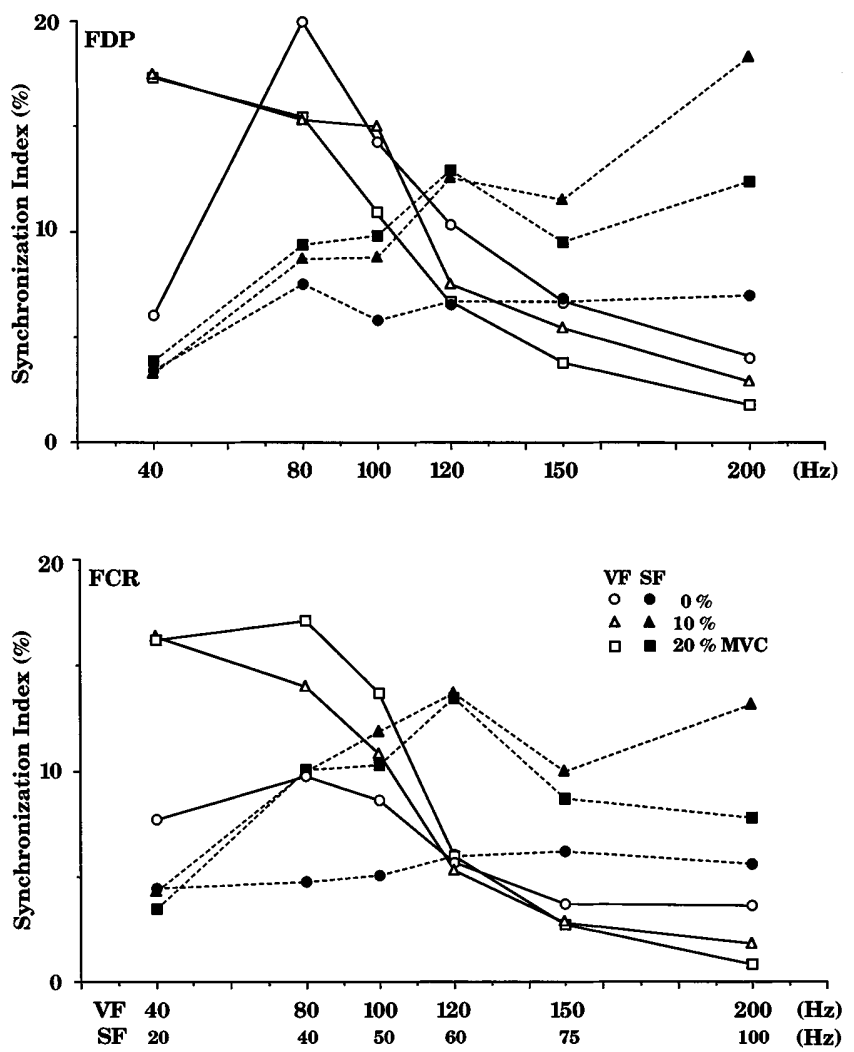

Fig. 3 Influence of vibration frequency and initial muscle contraction on the normalized indices of synchronization $\left(\mathrm{SYNC}_{\mathrm{VF}}\right.$, solid lines; $\mathrm{SYNC}_{\mathrm{SF}}$, dashed lines) for the FDP (upper panel) and the FCR (lower panel). Each data point represents the average over 10 subjects. Synchronization of motor units is enhanced by the initial contractions; harmonic synchronization decreases while subharmonic synchronization increases as vibration frequency increases (see text for detailed description). ( $V F$ Vibration frequency, $S F$ subharmonic of vibration frequency) 
that was correlated with vibration frequency was higher for the FDP than for the FCR $(P<0.001)$. This difference was most predominant at rest (Fig. 3 upper and lower panels) for vibration frequencies of $80-150 \mathrm{~Hz}$ $(P<0.05)$. There was no significant difference observed at the subharmonic frequency between the two muscles $(P=0.28)$. Furthermore, it can be seen in Fig. 3 that the "cut off" frequency is slightly higher for the FDP than for the FCR for initial contractions up to $10 \%$ MVC.

\section{Moderate exertion for a long duration}

The results describing changes in grip force, rmsEMG and the mean frequency of the PDS as a function of time are illustrated in Fig. 4 for the FDP muscle. Each data point corresponds to the analysis of the preceding $15 \mathrm{~s}$, averaged across subjects. Without vibration (control), a non-significant $(P=0.22)$ decay of the grip force with time was observed. When the moderate muscle contraction $(20 \% \mathrm{MVC})$ was maintained during vibration, a significant $(P<0.01)$ progressive decrease in grip force occurred. An abrupt decay of the grip (first $30 \mathrm{~s}$ ) was followed by a decay at a rate higher than that under the control situation. In both cases the respective rmsEMGs and mean frequency of the FDP did not vary significantly over time $(P>0.1)$; however, after $5 \mathrm{~min}$ the EMGs tended to increase (not statistically significant) while the grip forces remained constant. Peaks that were
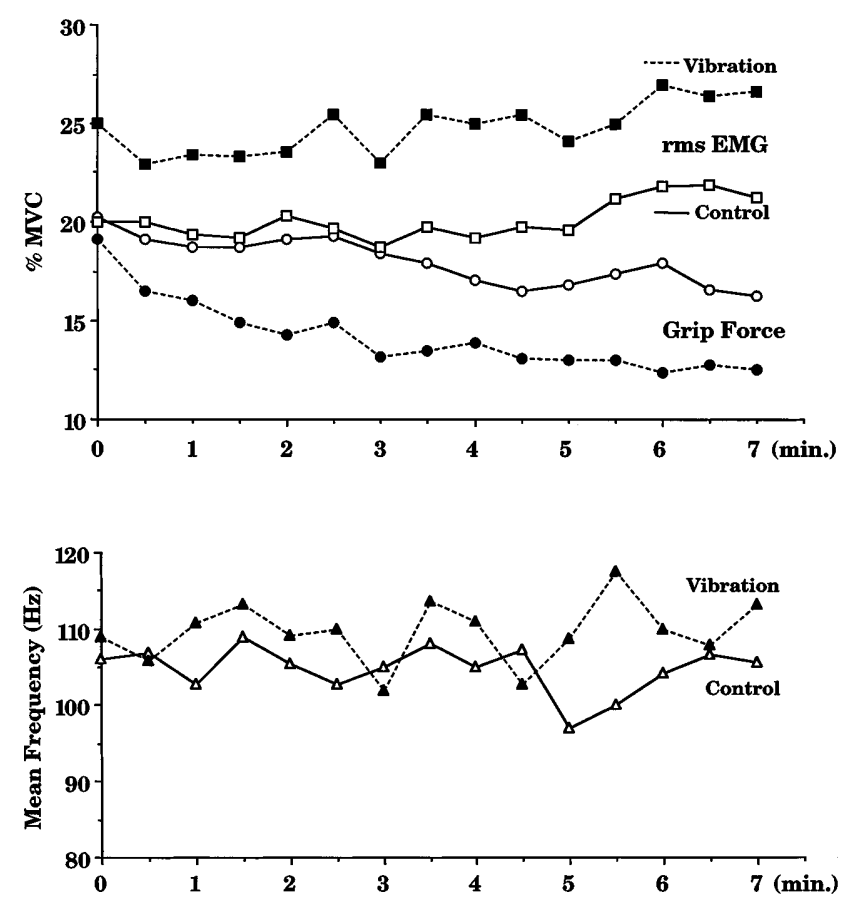

Fig. 4 Effects of vibration on moderate grip exertion of long duration. Grip force (upper panel) decays during vibration while the EMG rms increases and the mean frequency of the EMG power spectra (lower panel) does not vary significantly as a function of time. Data averaged over 5 subjects correlated with the vibration frequency were still present at the end of the vibration period.

\section{Discussion}

The main results showed that vibration-induced increases in EMG activity and the degree of motor unit synchronization were dependent on the vibration frequency and the initial contraction level. These results correspond to the "steady-state" phase of the TVR analyzed during the last $15 \mathrm{~s}$ of the vibration period.

To help interpret the present data, a brief account of previous results (Park and Martin 1993) is warranted. The strength of the TVR, as measured by $\triangle E M G$, increased with initial contraction up to $10 \% \mathrm{MVC}$ for the FDP and FCR, while an increase with vibration amplitude was observed only for the FCR. In addition, the strength of the TVR varies with vibration frequency for both muscles but in slightly differing ways. For the FDP, the TVR increased with vibration frequency up to $100 \mathrm{~Hz}$, increased at a slower rate up to $150 \mathrm{~Hz}$ and then decreased beyond that, with the exception of $10 \%$ MVC. In this latter condition the TVR magnitude was less at $120 \mathrm{~Hz}$ than at 100 or $150 \mathrm{~Hz}$. For the FCR, the TVR increased with frequency up to $100 \mathrm{~Hz}$, then decreased (Fig. 5). Hence, the magnitude of the changes of the TVR as a function of vibration frequency was dependent on the initial contraction and the muscle considered. The grip force did not vary significantly during vibration.

\section{Synchronization}

The "frequency response" of the TVR seems to be highly correlated with a motoneuron recruitment/derecruitment
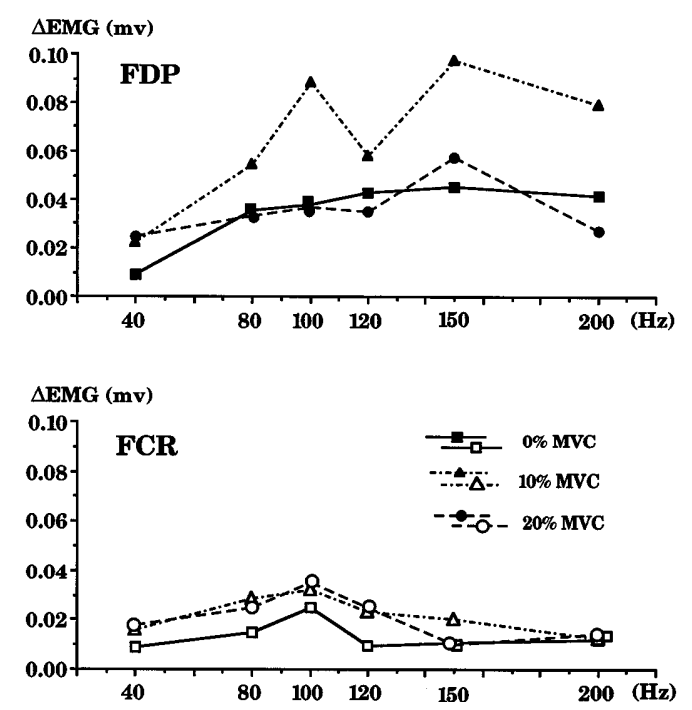

Fig. 5 Effect of vibration frequency on average (10 subjects) tonic vibration reflex magnitude $(\triangle E M G)$ of the FDP (upper panel) and FCR (lower panel) for increasing initial muscle contractions (resting, $10 \%$ and $20 \% \mathrm{MVC}$ ). (MVC Maximal voluntary contraction) 
process resulting from the "frequency response" of the primary spindle endings. Indeed, these receptors can respond in 1:1 synchrony up to about $100-150 \mathrm{~Hz}$ (Burke et al. 1976a; Roll et al. 1989); beyond this "cut-off" frequency most receptors start to respond at subharmonic frequencies and then at random (Roll et al. 1989). At low vibration frequencies $(\leq 100 \mathrm{~Hz})$, first harmonic synchronization was predominant and the magnitude of the TVR increased with vibration frequency. First, we may assume that almost all Ia afferents were recruited by the vibratory stimulus (Roll et al. 1989) and that the recruitment did not vary significantly with frequency since the displacement amplitude was the same at all frequencies (Martin et al. 1986). Second, we have previously demonstrated that below $100 \mathrm{~Hz}$ the inhibition of the monosynaptic pathway does not vary with vibration frequency at a constant displacement amplitude for the soleus muscle (Martin et al. 1986). Hence, an increase in the TVR in this frequency range results principally from an increase in motoneuron depolarization with the firing frequency of Ia afferents, which leads to a recruitment of motor units of increasing threshold.

At high vibration frequencies $(\geq 100-150 \mathrm{~Hz})$ most Ia afferents start to "misbehave", lose the response in terms of 1:1 synchrony (Burke et al. 1976a; Roll et al. 1989) and subharmonic synchronization is predominant. This change in behavior is illustrated in Fig. 2. Thus, a derecruitment process affecting the motoneurons in a reverse order is likely to occur, following Henneman's principle (Henneman et al. 1965). The declining excitatory drive of the harmonic response of Ia fibers is likely to be balanced by the increase in both the subharmonic response (Fig. 2) and the asynchronous activity until these components lose their own influences as the frequency increases beyond $200 \mathrm{~Hz}$. This latter effect, due to the decay in firing frequency and in the number of responsive fibers (Roll et al. 1989), is illustrated in part by a stabilization or a decline in the subharmonic component beyond $120 \mathrm{~Hz}$ (Fig. 2).

Aside from the intrinsic bandwidth properties of the primary endings, the weakening of the Ia fiber response with the increase of vibration frequency may also have mechanical and reflex origins. First, the visco-elastic muscle-tendon system behaves like a low-pass filter in the high frequency range and attenuates vibration transmissibility to the spindles. This filtering results in a decrease in Ia-afferent driving. Second, the strength of facilitatory inputs to the $\gamma$-system from several afferents (Appelberg et al. 1985; Johansson et al. 1989; Sojka et al. 1991) mediating respectively the vibration-induced activity of skin, joint and secondary endings also fades away as vibration frequency increases, due to the low "cut off" frequency of the aforementioned mechanoreceptors (Burke et al. 1976a; Roll et al. 1989). Hence, the decrease of the gain of the nested positive reflex loop (group II, joint and skin afferents $\rightarrow \gamma$-motoneurons $\rightarrow$ Ia-afferents) affects the sensitivity of primary endings and contributes to the decay of vibration-induced synchronization of $\alpha$-motoneurons and to their derecruitment.
An alternative explanation can be proposed. The roughly "inverted U" shape of the TVR as a function of vibration frequency may suggest a resonance of the musculo-tendinous system, which would increase the stretch of the spindle endings and their response as a consequence. Hence, under the assumption of a nearmaximal driving of Ia-afferents indicated above, potentiation of the TVR would essentially result from an activation of the less sensitive secondary endings (Roll et al. 1989; Cordo et al. 1993). In such a case or even if more Ia fibers could respond, we should not observe a decrease in $\mathrm{SYNC}_{\mathrm{VF}}$ for frequencies below the presumed resonance. Although a mechanical resonance cannot be ruled out, it probably does not play a significant role in the present context.

\section{Localized muscular fatigue}

During the whole vibration period, the EMG activity remains $25 \%$ higher than the control value. The initial change in force exertion, paralleled by a decrease in EMG (Fig. 4; at $t=0.5 \mathrm{~min}$ ), is most probably due to the alteration of force perception induced by localized tendon vibration (Cafarelli and Kosta 1981). Nevertheless, the continuous decrease in force that follows, not paralleled by a decrease in EMG but rather concurrent with a constant EMG level exhibiting no change in a mean frequency of the power spectra, suggests a vibration-forced driving of the motor units leading to an impairment of excitation-contraction coupling (for review see Stephenson et al. 1992). This hypothesis stems from the organization of motor unit reflex patterns and possible fatigue mechanisms.

First, in the context of an initial contraction $(20 \%$ MVC) and a high vibration frequency $(100 \mathrm{~Hz})$, the steady state of the TVR is certainly powered by a large proportion of high-threshold motor units activated predominantly by polysynaptic pathways (Romaiguère et al. 1991; Lebedev and Polyakov 1992; Burke et al. 1976b), with a lesser proportion of slow, low-threshold motor units activated monosynaptically (Romaiguère et al. 1991). Also Romaiguère et al. (1991) have shown that the polysynaptic components can be phase-locked to the vibration cycle. As motor unit fatigue resistance decreases inversely with the recruitment threshold (Stephens and Usherwood 1977), it seems reasonable to expect that muscle fatigue develops at a higher rate under vibration.

Second, the present results suggest that fatigue compensation mechanisms of neurological origin, e.g., increase in the discharge rate and recruitment of additional motor units (Bigland-Ritchie et al. 1986; Maton and Game 1989), are hindered. On the one hand, the driving process induced by vibration forces the recruited motor unit to discharge at a relatively high rate (Romaiguère et al. 1991), which precipitates fatigue. On the other hand, most of the pool of motor units that would have been activated by the adjustment of the motor command 
for the assumed level of effort are already driven by the vibration-triggered mechanisms. Recent studies (Bongiovanni and Hagbarth 1990; Bongiovanni et al. 1990) demonstrate that vibration is indeed able to re-recruit and temporarily increase the firing rate of motor units fatigued by a maximal effort, with a more pronounced facilitatory effect on high-threshold than low-threshold units. Such results suggest that vibration has a greater driving power than fatigue compensatory mechanisms. This power is certainly enhanced by the fusimotordriven feedback (see nested positive feedback above). In the absence of vibration, a decrease in the gain of this feedback is thought to contribute to a decrease of the motor output (for review of the "sensory feedback hypothesis" and fatigue see Enoka and Stuart 1992). The forced driving of motor units is supported by the fact that synchronization observed from individual recordings did not deteriorate significantly over the time period considered here. This latter effect was also observed by Lebedev and Polyakov (1992). Hence, although a metabolic origin cannot be excluded, myofibrillar fatigue and/or failure of activation of the contractile system, reported by Edman and Lou (1992), are mechanisms that may take place under continuous driving of the motor units and explain the weakening of force despite an increased EMG level.

To conclude, this study indicates, first, that the index of synchronization could be used to determine the relative sensitivity of the Ia-afferent feedback. Second, high-frequency vibration $(>150 \mathrm{~Hz})$ tends to induce less muscle/tendon stress and motor unit synchronization. Third, the vibration-induced stress reaches its maximum at a moderate level of force exertion. These results find application in power hand tool design since the TVR also exists when they are used (Radwin et al. 1987). As transmissibility of vibration to the forearm system is limited beyond $100 \mathrm{~Hz}$, tools generating low-intensity, high-frequency vibration $(>150 \mathrm{~Hz})$ should be preferred, provided that mechanical resonance does not occur.

Acknowledgements This work was supported by grants from the National Institute for Occupational Safety and Health (no. 5RO1OHO2967) and from Rackham School of Graduate Studies of The University of Michigan.

\section{References}

Appelberg B, Johansson HP, Sojka P (1985) Fusimotor reflexes in triceps surae muscle elicited by stretch of muscles in the contralateral hind limb of the cat. J Physiol (Lond) 363:403-417

Bigland-Ritchie B, Cafarelli E, Vøllestad NK (1986) Fatigue of submaximal static contraction. Acta Physiol Scand Suppl 556:137-148

Bongiovanni LG, Hagbarth KE (1990) Tonic vibration reflex elicited during fatigue from maximal voluntary contractions in man. J Physiol (Lond) 423:1-14

Bongiovanni LG, Hagbarth KE, Stjernberg L (1990) Prolonged muscle vibration reducing motor output in maximal voluntary contractions in man. J Physiol (Lond) 423:15-26
Burke D, Hagbarth KE, Lofstedt L, Wallin BG (1976a) The response of human muscle spindle endings to vibration during isometric contraction. J Physiol (Lond) 261:695-711

Burke D, Rymer WZ, Walsh HV (1976b) Relative strength of synaptic inputs from short latency pathways to motor units of defined type in cat medial gastrocnemius. Neurophysiology 39:447-458

Cafarelli E, Kosta E (1981) Effects of vibration on static force sensation in man. Exp Neurol 74:331-340

Cordo P, Gandevia SC, Hales JP, Burke D, Laird G (1993) Force and displacement controlled tendon vibration in humans. Electroencephalogr Clin Neurophysiol 89:45-53

De Gail P, Lance JW, Neilson PD (1966) Differential effects on tonic and phasic reflex mechanisms produced by vibration of muscles in man. J Neurol Neurosurg Psychiatry 29:1-11

Desmedt JE, Godaux E (1980) The tonic vibration reflex and the vibration paradox in limb and jaw muscles in man. In: Desmedt JE (Ed) Spinal and supraspinal mechanisms of voluntary motor control and locomotion. Karger, Basel

Edman KA, Lou F (1992) Myofibrilar fatigue versus failure of activation during repetitive stimulation of frog muscle fibers. J Physiol (Lond) 457:655-673

Enoka RM, Stuart DG (1992) Neurobiology of muscle fatigue. J Appl Physiol 72:1631-1647

Hagbarth KE, Eklund G (1965) Motor effects of vibratory muscle stimuli in man. In: Granit R (ed) Muscular afferents and motor control. Proceedings of the First Nobel Symposium. Almqvist and Wiksell, Stockholm

Henneman E, Somjen G, Carpenter DO (1965) Excitability and inhibitability of motoneurons of different sizes. J Neurophysiol 28:599-620

Homma S, Kanda K, Watanabe S (1972) Integral pattern of coding during tonic vibration reflex. In: Somjen GG (ed) Neurophysiology studied in Man. Experta Medica, Amsterdam, pp 345349

Hori Y, Higara K, Watanabe S (1989) The effects of thiamyal sodium on the tonic vibration reflex in man. Brain Res 497:291295

Johansson H, Sjolander P, Sojka P, Wadell I (1989) Reflex actions on the muscle-spindle systems of muscles acting at the knee joint elicited by stretch of the posterior cruciate ligament. Neuro-Orthop 8:9-21

Lebedev MA, Polyakov AV (1992) Analysis of surface EMG of human soleus muscle subjected to vibration. J Electromyogr Kinesiol 2:1-10

Martin BJ, Roll JP, Gauthier GM (1986) Inhibitory effects of combined agonist and antagonist muscle vibration on H-reflex in man. Aviat Space Environ Med 57:681-687

Maton B, Game D (1989) The fatiguability of two agonist muscles in human isometric voluntary submaximal contraction: an EMG study. Eur J Applied Physiol 58:369-374

Matthews PBC (1966) The reflex excitation of the soleus muscle of the decerebrate cat caused by vibration applied to its tendon. J Physiol (Lond) 184:450-472

Park HS, Martin B (1993) Contribution of the tonic vibration reflex to muscle stress and muscle fatigue. Scand J Work Environ Health 19:35-42

Pyykkö I, Färkkilä M, Toivanen J, Korhonenand $\mathrm{O}$, Hyvarynen J (1976) Transmission of vibration in the hand-arm system with special reference to changes in compression force and acceleration. Scand J Work Environ Health 2:87-95

Radwin RG, Armstrong TJ, Chaffin DB (1987) Power hand tool vibration effects on grip exertions. Ergonomics 30:833-855

Reynolds D (1977) Hand-arm vibration: a review of 3 years' research. In: Wasserman DE, Taylor W (eds) Proceedings of the International Occupational Hand-Arm Vibration Conference. DHEW Publications no. 77-170:99-128

Roll JP, Gilhodes JC, Tardy-Gervet MF (1980) Effets perceptifs et moteurs des vibrations musculaires chez l'homme normal. Mise en évidence d'une réponse des muscles antagonistes. Arch Ital Biol 118:51-71 
Roll JP, Vedel JP, Ribot E (1989) Alteration of proprioceptive messages induced by tendon vibration in man: a microneurographic study. Exp Brain Res 76:213-222

Romaiguère P, Vedel JP, Azulay JP, Pagni S (1991) Differential activation of motor units in the wrist extensor muscles during the tonic vibration reflex in man. J Physiol (Lond) 444:645-667

Sojka P, Sjolander P, Johansson H, Djupsjöbacka M (1991) Influence from stretch-sensitive receptors in the collateral ligaments of the knee joint on the muscle-spindle systems of flexor and extensor muscles. Neurosci Res 11:55-62
Stephens JA, Usherwood TP (1977) The mechanical properties of human motor units with special reference to their fatiguability and recruitment threshold. Brain Res 125:91-97

Stephenson DG, Lamb GD, Stephenson GMM, Fryer MW (1992) Mechanisms of excitation-contraction coupling relevant to skeletal muscles. In: Gandevia SG, Enoka R, McComas AJ, Stuart DG, Thomas CK (eds) Fatigue neural and muscular mechanisms, advances in experimental medicine and biology, vol. 384. Plenum, New York, pp 45-68 